\title{
Nuclear lamina strain states revealed by intermolecular force biosensor
}

Brooke E. Danielsson ${ }^{1}$, Bobin George Abraham², Elina Mäntylä2*, Jolene I. Cabe1, Carl R Mayer $^{1}$, Anna Rekonen², Frans Ek², Daniel E. Conway ${ }^{1 \uparrow}$, Teemu O Ihalainen ${ }^{2 \uparrow}$

* Contributed equally

ๆ Co-corresponding authors: dconway@vcu.edu, teemu.ihalainen@tuni.fi

1) Department of Biomedical Engineering, Virginia Commonwealth University, Richmond, Virginia, USA

2) BioMediTech, Faculty of Medicine and Health Technology, Tampere University, Tampere, Finland

\begin{abstract}
We developed an intramolecular FRET-based strain biosensor using nanobodies capable of measuring the mechanical strain of lamin filaments. Using this sensor, we were able to show that the nuclear lamina is subjected to significant strains. These strains are dependent on nuclear volume, actomyosin contractility, functional LINC complex, chromatin condensation state, cell cycle, and EMT. Overall, we demonstrate that nanobody-based approach allows construction of novel force biosensors for mechanobiology studies.
\end{abstract}

\section{Main Text}

Mechanical forces are important co-regulators of many physiological processes[1]. In addition to mechanotransduction at the surface of the cell, the cytoskeleton also allows transmission of forces throughout the cell, including onto and within the nucleus[2]. Thus, the nucleus has emerged as a putative mechanosensitive structure. The lamin proteins, together with Linker of Nucleoskeleton and Cytoskeleton (LINC) complex, consisting of nesprin and SUN proteins, is the principal structure that connects the nucleus to the cytoskeleton[3]. These connections enable a mechanotransmission pathway, where mechanical stress can be transduced outside-in and insideout between the cell surface and the nucleus via the cytoskeleton[4].

The lamina is approximately $15 \mathrm{~nm}$ thick protein meshwork, formed mainly from flexible $\sim 400$ $\mathrm{nm}$ long A-type and B-type lamin filaments[5], [6]. Large parts of the chromatin are either stably or dynamically tethered to the nuclear lamina and this tethering has been shown to regulate gene 
expression[5]. Especially A-type lamin proteins, lamin A/C, are also located throughout the nucleoplasm. Although less characterized, these intranuclear or nucleoplasmic lamins bind to chromatin and have been shown to regulate chromatin accessibility and spatial chromatin organization[7].

Similar to other intermediate filament proteins, in vitro experiments of purified nuclear lamins have shown that these proteins are able to withstand large mechanical forces, exhibit deformation under mechanical loading, and exhibit strain-stiffening behavior[8]. To study the mechanical loading of lamins in vivo, we sought to develop a biosensor for lamin A/C. Prior force biosensor design strategies consisted of chimeric proteins in which FRET-force module (a FRET pair separated by a strain-sensitive peptide) was inserted in the middle of the protein[1]. These intramolecular force sensors have been successfully used for proteins at focal adhesions, cell-cell adhesions, and the nuclear LINC complexes[9], [10][11][12]. However, concerns remain regarding how internal insertion of a large FRET-force module $(\sim 50 \mathrm{kDa})$ could alter the biological functions of the protein. This may be especially important in the context of filamentous proteins, such as the nuclear lamina, where altered lamin proteins could disrupt or impair the oligomerization and/or polymerization of filamentous structures. Here we report a new intermolecular strain sensor concept and use it for measuring mechanical forces on lamin A/C. We developed a lamin A/C strain sensor (Lamin-SS), which consists of an existing FRET force module, known as TSmod[13] with N- and C-terminal lamin A nanobodies[14] (Fig. 1a). A straininsensitive truncated control sensor (Lamin-SS-T), containing only an N-terminal lamin A nanobody, was also developed (Fig. 1a). The fluorescence of both sensors was strongly correlated to lamin A/C immunostaining (Fig. 1b), indicating strong localization to the nuclear lamina. Fluorescence recovery after photobleaching (FRAP) experiments showed that both nanobodies of Lamin-SS can simultaneously bind the nuclear lamina and the dual nanobody binding extends the sensor residence time in the lamina when compared to single nanobody (Supplemental Fig. 1, 2). Lamin-SS exhibited a large FRET decrease as compared to Lamin-SS-T (Fig. 1c) indicating an increased distance (strain) between the FRET pair for Lamin-SS. Fluorescence-lifetime imaging microscopy (FLIM) also showed reduced FRET (measured as increased lifetime) for Lamin-SS, as compared to Lamin-SS-T (Fig. 1d). Intriguingly both methods showed similar levels of FRET at the nuclear perimeter and in the nucleoplasm, suggesting similar levels of strain for lamin A in each region of the nucleus. Loss of localization at the nuclear rim and higher FRET for Lamin-SS was observed in lamin KO cells (Supplemental Fig 3), showing that changes in Lamin-SS FRET are dependent on A-type lamins. Additionally, we observed a rapid increase in Lamin-SS FRET ratio during nuclear strain relaxation induced by higher osmolarity of the medium (Fig. 1e and Supplemental Fig. 4). These data demonstrate that Lamin-SS FRET is inversely related to lamin A strain.

As an additional measure of lamin A/C forces, as well as a proof of concept for the utility of other nanobody based intermolecular sensors, we developed a second sensor to measure mechanical tension between histone 2A/2B (H2A/2B) and lamin A/C, Lamin-histone-SS (Supplemental Fig. 
5). This sensor exhibited reduced FRET in comparison to a truncated sensor Lamin-histone-SS-T and FRET was affected by depletion of A-type lamins (Supplemental Fig. 3, 5). These results indicates that mechanical forces can be transduced between chromatin and lamin A.

Next, we sought to identify key cytosolic components which regulate lamin A/C strain. Using Lamin-SS we observed that actomyosin inhibition with ROCK-pathway inhibitor (Y-27632) resulted in decreased strain (increased FRET) (Fig. 2a, b). Additionally, the sensor was successfully used to temporally analyze lamin A/C strain changes during actomyosin inhibition (Fig. 2c, d). Treatment with actin fiber depolymerizing agent (cytochalasin D) also reduced lamin A/C strain (Supplemental Fig. 6). Thus, the intact actin cytoskeleton and myosin contractility regulate lamin A/C strain. Next, we sought to understand the role of the LINC complex in lamin strains. Disruption of the LINC complex using a dominant negative nesprin construct (DN-KASH) modestly reduced lamin A/C strain (Fig. 2e, f).

Additionally, we sought to understand how changes in chromatin structure affect lamin A/C strain. When chromatin was decondensed by using a histone deacetylase inhibitor (TSA), we detected a significantly decreased Lamin A/C strain (Fig. 2g, h). TSA was shown to significantly increase H3K27 acetylation without altering the organization of A-type lamins, detected by labeling specific lamin A/C epitopes (Supplemental Fig. 7). TSA treatment also did not affect Lamin-SS binding, as measured by FRAP (Supplemental Fig. 8). Chromatin condensation, achieved via histone trimethyl demethylase inhibitor (methylstat), exhibited a small, but non-significant increase in lamin A/C strain. Similarly, to TSA treatment, methylstat did not affect the A-type lamin organization (Supplemental Fig. 9).

Finally, we sought to examine how perturbations in the cell cycle and cell phenotype regulate lamin A/C strain. When cells were arrested to early S-phase by treatment with DNA polymerase $\alpha$ inhibitor (aphidicolin), we detected decreased lamin A/C strain (Fig. 2i). Induction of epithelial to mesenchymal transition (EMT), using TGF- $\beta$, also resulted in decreased lamin A/C strain (Fig. $2 \mathrm{j})$.

This technical advancement provides significant insight into nuclear mechanics, by providing the first direct measurements of nuclear lamina strain. Lamin A/C strain, presumably the result of tensile and compressive mechanical forces, is dynamic and influenced in both an outside-in (actomyosin, LINC complex) and inside-out (chromatin) manner. Additionally, we show that intranuclear lamins also experience significant levels of strain, providing additional evidence that nucleoplasmic lamins are an important structural element of the nucleus. This work demonstrates the potential for nanobody-based biosensors to be further utilized to measure mechanical strains between proteins. 


\section{Acknowledgments}

We thank Jan Lammerding and Alice Varlet for thoughtful discussions and Heidi Peussa for her help with the LMNA KO cells. The authors acknowledge the Biocenter Finland (BF), Tampere Imaging Facility (TIF), and the VCU Nanomaterials Characterization Core (NCC) microscopy services. In addition, we wish to acknowledge Tampere University Virus Facility and Eric Dufour for the help in sgRNA design, Flow Cytometry Facility and Laura Kummola for the services and Light Microscopy Unit supported by HiLIFE and BE, Institute of Biotechnology, University of Helsinki, for the FLIM imaging. This project was funded in part by a National Science Foundation Graduate Research Fellowship (to B.E.D.), National Science Foundation CAREER award CMMI 1653299 (to D.E.C.), National Institute of Health award R35 GM119617 (to D.E.C.), as well as Academy of Finland under the award numbers 308315, 314106, 33520 (to T.O.I.) and 332615 (to E.M). 


\section{Figure legends}

\section{Figure 1. Development and characterization of the FRET based lamin A/C strain sensor.}

a, Schematic representation of the FRET based lamin A/C strain sensor (Lamin-SS), truncated control sensor (Lamin-SS-T) and the working mechanism of the strain sensing. b, Laser scanning confocal microscopy images (Airy-scan, single sections) of immunolabeled lamin $\mathrm{A} / \mathrm{C}$ together with the expressed Lamin-SS or Lamin-SS-T sensor along with corresponding fluorescence lineprofiles. Scale bars, $5 \mu \mathrm{m}$. c, FRET efficiency images and quantified FRET efficiency of LaminSS and Lamin-SS-T sensors. The plots represent the median \pm SEM of individual image fields. Lamin-SS had a median FRET efficiency of $17 \%$ compared to Lamin-SS-T with 40\%. (total $\mathrm{n}=$ 10 fields from 3 replicates) Scale bar $20 \mu \mathrm{m}$. Unpaired Student's t-tests $(* * * p<0.0001)$. d, Donor fluorescence lifetimes of free donor (mTFP1), Lamin-SS and Lamin-SS-T along with FLIM images of Lamin-SS and Lamin-SS-T expressing cells $(n=36, n=43$ and $n=42$ cells from 2 replicates). Scale bar $20 \mu \mathrm{m}$. e, Apparent FRET efficiency images of osmotically stressed LaminSS expressing cells together with quantified apparent FRET efficiency. Plot represents the data from a single measurement (total $n=304$ cells from 3 replicates). Lamin-SS had a mean FRET ratio $\pm \mathrm{SEM}$ of $0.064 \pm 0.001$ in MEM compared to $0.114 \pm 0.001$ in hyperosmotic conditions (MEM $+250 \mathrm{mM}$ sucrose, $15 \mathrm{~min})$. Paired Student's t-test $(* * * \mathrm{p}<0.0001)$. Scale bars, $20 \mu \mathrm{m}$.

Figure 2. The effect of cellular force transduction and chromatin organization on lamin $\mathrm{A} / \mathrm{C}$ strain.

a, Cellular contractility was reduced by ROCK-inhibition (Y-27632, $50 \mu \mathrm{M}, 1 \mathrm{~h}$ ). FRET efficiency images of Lamin-SS and Lamin-SS-T after inhibition. Scale bars, $20 \mu \mathrm{m}$. b, Quantified FRET efficiency of Lamin-SS and Lamin-SS-T sensors after ROCK-inhibition. FRET efficiency of Lamin-SS was $22 \%$ with Y-27632 and $14 \%$ without treatment. In the same conditions FRET efficiencies were $39 \%$ and $40 \%$ with Lamin-SS-T, respectively. ( $\mathrm{n}=10$ fields from 3 replicates), Unpaired Student's t-test $(* * \mathrm{p}<0.01)$. c, Live-cell time lapse apparent FRET efficiency imaging of Lamin-SS during ROCK-inhibition (Y-27632, $50 \mu \mathrm{M}$ ). Scale bar $10 \mu \mathrm{m}$. d, Quantified relative change (mean \pm SEM) in Lamin-SS FRET ratio during ROCK-inhibition time lapse imaging $(\mathrm{n}=$ 152 cells from 2 replicates, black and grey). E, LINC-complexes were disrupted by expressing dominant-negative KASH construct (induction for 24h). FRET efficiency images of Lamin-SS and Lamin-SS-T after LINC disruption. Scale bars, $20 \mu \mathrm{m}$. f, Quantified FRET efficiency of Lamin-SS and Lamin-SS-T after LINC-complex disruption. FRET efficiency of Lamin-SS was 28 $\%$ with DN-KASH expression, $22 \%$ without DN-KASH expression. The efficiencies were $53 \%$ and $54 \%$ with Lamin-SS-T, respectively ( $\mathrm{n}=10$ from 3 replicates). Unpaired Student's t-test (* $\mathrm{p}<0.05$ ). g, Chromatin was relaxed by using histone deacetylase inhibitor (TSA, $200 \mathrm{nM}, 4 \mathrm{~h}$ ). Images and quantification of Lamin-SS and Lamin-SS-T FRET efficiency after treatment of the cells. FRET efficiency of Lamin-SS was $21 \%$ after TSA treatment, and $15 \%$ without TSA. The 
efficiencies were $36 \%$ and $37 \%$ with Lamin-SS-T, respectively ( $\mathrm{n}=10$ from 3 replicates). Unpaired Student's t-test $(* * * \mathrm{p}<0.0001)$. Scale bars $20 \mu \mathrm{m}$. h, Chromatin was condensed by using histone demethylase inhibitor (methylstat, $2.5 \mu \mathrm{M}, 48 \mathrm{~h}$ ). Images and quantification of Lamin-SS and Lamin-SS-T FRET efficiency after treatment of the cells. FRET efficiency of Lamin-SS was $14.5 \%$ after methylstat treatment, $18 \%$ without methylstat. The efficiencies were $39 \%$ and $40 \%$ with Lamin-SS-T, respectively. ( $n=10$ from 3 replicates). Unpaired Student's t-test. Scale bars $20 \mu \mathrm{m}$. i, Cells were synchronized to early S-phase by blocking DNA polymerase (Aphidicolin, 3 $\mu \mathrm{g} / \mathrm{mL}, 24 \mathrm{~h}$ ). Images and quantification of Lamin-SS and Lamin-SS-T FRET efficiency after cell cycle synchronization. FRET efficiency of Lamin-SS was $21 \%$ after Aphidicolin treatment, $16 \%$ without Aphidicolin. The efficiencies were $39 \%$ and $40 \%$ with Lamin-SS-T, respectively $(n=10$ from 3 replicates). Unpaired Student's t-test $(* \mathrm{p}<0.05)$. Scale bars, $20 \mu \mathrm{m}$. j, EMT was induced by treating cells with a growth factor (TGF- $\beta 1,2 \mathrm{ng} / \mathrm{mL}, 24 \mathrm{~h}$ ). Images and quantification of Lamin-SS and Lamin-SS-T FRET efficiency after EMT induction. FRET efficiency of Lamin-SS was $22 \%$ after TGF- $\beta 1$ treatment, $17 \%$ without TGF- $\beta 1$. The efficiencies were $39 \%$ and $39 \%$ with Lamin-SS-T, respectively ( $\mathrm{n}=10$ from 3 replicates). Unpaired Student's t-test $(* \mathrm{p}<0.05)$. Scale bars, $20 \mu \mathrm{m}$. 


\section{Supplemental text}

\section{Materials and Methods}

\section{Sensor Design}

A sensor to measure mechanical forces on the nuclear lamins was designed using an existing lamin A nanobody. The nanobody was previously developed by Rothbauer et al[14] and is currently commercially distributed as a "chromobody", consisting of the nanobody tagged with GFP, by Chromotek (Planegg-Martinsried, Germany). The sensor is designed such that an existing FRETforce biosensor, known as TSmod[15] is flanked on either side by the lamin A nanobody $\mathrm{V}_{\mathrm{H}} \mathrm{H}$ sequence (Fig. 1a). To ensure nuclear localization of the protein a c-myc NLS was inserted between each nanobody and TSmod. Additionally, the C-terminal lamin A nanobody was designed by using the reverse sequence of the $\mathrm{V}_{\mathrm{HH}}$ for orientation of the nanobody outwards from TSmod. The entire sequence of the nanobody-TSmod-reverse nanobody was synthetically cloned by GeneArt (Thermo Fisher Scientific) into pcDNA 3.3. The plasmid is available through Addgene (plasmid\# 178641). A control force-insensitive lamin sensor, consisting of only one nanobody attached to TSmod was also developed and is available through Addgene (plasmid\# 178642).

A second sensor was developed designed to measure forces between the nuclear lamina and histones. This sensor consists of a nanobody which binds to the Histone H2A-H2B heterodimer that was previously developed by[16] and is also commercially distributed as a "chromobody" by Chromotek. The sensor was designed similarly to the lamin sensor, with the N-terminal lamin A sensor being replaced with the histone nanobody (histone nanobody-TSmod-reverse lamin A nanobody). This sensor was also synthetically cloned by GeneArt and is available through Addgene (plasmid\# 178643). A control force-insensitive histone sensor, consisting of only the histone nanobody attached to TSmod was also developed and is available through Addgene (plasmid\# 178644).

\section{Cells}

Madin-Darby canine kidney cells (MDCK II) were used in all studies and maintained in high glucose DMEM (Thermo Fisher Scientific) to which was added 10\% fetal bovine serum (Thermo Fisher Scientific) and $1 \%$ penicillin/streptomycin (Thermo Fisher Scientific) under standard cell culture conditions. To generate stable cell lines MDCKs were transfected with the TSmod and selected using G418. For the DN-KASH experiments, DN-KASH inducible Lamin-SS cells and DN KASH inducible Lamin-SS-t cells were made into stable cell lines. To generate a system for doxycycline-inducible DN-KASH Lamin-SS cells and doxycycline-inducible DN-KASH Lamin- 
SS-T cells, the previously established doxycycline-inducible DN-KASH MDCK cells[17] were electroporated with Lamin-SS pcDNA and Lamin-SS-T pcDNA separately. Cells expressing both DN-KASH and Lamin-SS/SS-T were extracted with cloning rings and were clonally expanded.

\section{Establishment of LMNA knockout with CRISPR/Cas9}

To generate a pre-LMNA knockout (KO) MDCK II cell line with CRISPR/Cas9, single guide RNAs (sgRNAs) were custom made from Invitrogen backbone from their LentiArray ${ }^{\mathrm{TM}}$ Human CRISPR Library and designed against LMNA1 gene N-terminus in CanFam 3.1 reference genome (https://www.ncbi.nlm.nih.gov/nuccore/NM_001287151.1, GeneID: 480124) with an online guide design tool. LMNA target sequence: atggagac cccgtcccag cggcgegcca cccgtagcgg ggcgcaggcc agctccacce cgctgtcgcc caccegcatc acceggctgc aggagaagga ggacctgcag gagctcaatg accgcctggc ggtctacatc gaccgtgtgc gctctctgga gacggagaac gcggggctgc gecttcgcat caccgagtcg. The sgRNA_LMNA_N1 nucleotide sequence was CACGGTCGATGTAGACCGCC (on-target locus chr7:-41719582). For expression, the sgRNA_LMNA_N1 (300 ng) and pCDNA3.1-dCas92xNLS-EGFP (gift from Eugene Yeo, \#74710, Addgene; http://n2t.net/addgene:74710) were transfected by using the Neon ${ }^{\mathrm{TM}}$ electroporation system $(1650 \mathrm{~V}, 20 \mathrm{~ms}, 1$ pulse; Thermo Fisher Scientific) followed by selection of GFP-positive cells with G418 $(0.25 \mathrm{mg} / \mathrm{mL}$, Merck) and FACS sorting (BD FACSAria Fusion, BD Biosciences)[18]. The success of the KO was determined via Western blot and further confirmed by immunostainings.

\section{Drug Treatments}

For actin depolymerization studies, Cytochalasin D (cat \# 11330, Caymen Chemical) was used at $10 \mu \mathrm{g} / \mathrm{mL}$ for $1 \mathrm{~h}$. To inhibit Rho A kinase, $50 \mu \mathrm{M}$ Y-27632 (cat \#72302, Stem Cell Technologies) was used for 1 hour prior to FRET imaging to reduce myosin activity. For EMT induction, recombinant human TGF- $\beta 1$ (R\&D systems) was used to induce EMT at a concentration of 2 $\mathrm{ng} / \mathrm{mL}$ for $24 \mathrm{~h}$. Modifications in DNA ultrastructure were done to condense or decondense chromatin with the use of $600 \mathrm{nM}$ trichostatin A (TSA) for $4 \mathrm{~h}$ (Cayman Chemical Company), to increase euchromatin, and $2.5 \mu \mathrm{M}$ methylstat (Sigma Aldrich) for $48 \mathrm{~h}$, to increase heterochromatin. For the cell cycle synchronization assay, Aphidicolin (cat \#57-361, Thermo Fisher Scientific) was used to block the cells in early S-phase, at $3 \mu \mathrm{g} / \mathrm{mL}$ for $24 \mathrm{~h}$.

\section{Immunofluorescence Staining of Lamins and Histones}

For fixed-cell experiments, cells were washed with PBS and fixed for $10 \mathrm{~min}$ at room temperature with $4 \%$ paraformaldehyde in PBS. After three washes with PBS, the cells were permeabilized 
for 10 min at room temperature with $0.2 \%$ Triton X-100 in PBS and blocked with $5 \%$ BSA for 1 $\mathrm{h}$ at room temperature. Cells were then incubated overnight at $4{ }^{\circ} \mathrm{C}$ or in room temperature with the primary $\mathrm{Ab}$ diluted in blocking solution. The following primary antibodies were used: antilamin A antibody (sc-7292, Santa Cruz Biotechnology), anti-mouse LA/C-C (131C3, ab8984, Abcam) anti-rabbit LA/C-rod (EP4520-16, ab133256, Abcam), or anti-Rabbit Histone H2A (cat \#12349, Cell Signaling Technology). Three more washes with PBS were then followed by incubation with the secondary Ab (Alexa Fluor 647-conjugated donkey anti-mouse IgG; Thermo Fisher Scientific) for 45 min followed by three additional PBS washes. Samples were stained with Hoechst 33342 (Thermo Fisher Scientific) and mounted with ProLong Gold antifade mountant (P36930, Thermo Fisher Scientific).

\section{Fluorescence Microscopy of Histones and Nuclear Lamina Organization}

Confluent non-treated or either trichostatin A (TSA, 4h, $600 \mathrm{nM})$ or methylstat $(48 \mathrm{~h}, 2.5 \mu \mathrm{M})$ treated MDCK II wt, MDCK II-TS or MDCK II-truncated mutant cells were analyzed to ensure the drug treatments used in FRET-experiments did not affect the nuclear lamina organization. To detect changes in nuclear lamina organization, ratiometric fluorescence immunoassay was performed on MDCKII wt, MDCK II cells stably expressing Lamin-SS or MDCK II cells stably expressing Lamin-SS-T immunostained against either lamin A/C N-terminus (LA/C-N, E1, sc376248, Santa Cruz Biotechnology, Texas, USA) and histone H3 lysine 27 acetylation (H3K27ac, ab4729, Abcam), or lamin A/C C-terminal (anti-mouse LAC/C-C, 131C3, ab8984, Abcam, Cambridge, UK) and lamin A/C rod domain (anti-rabbit LA/C-rod, EP4520-16, ab133256, Abcam) as described above. Imaging was done on a Nikon A1R+ laser scanning confocal mounted in Nikon Eclipse Ti2-E inverted microscope (Nikon Instruments, Tokyo, Japan). Nikon 60X/1.40 Apo DIC N2 oil immersion objective was used in the experiments. Solid state lasers with excitation wavelengths $488 \mathrm{~nm}, 561 \mathrm{~nm}$ and $640 \mathrm{~nm}$ were used in excitation. The emissions were collected with 525/50,540/30 and 595/50 bandpass filters, respectively. The laser intensities were adjusted to avoid photobleaching and the detector sensitivity was adjusted to optimize the image brightness and to avoid saturation. Laser powers and detector voltages were determined individually per treated antibody pair, and after the initial setting kept constant for each sample to allow ratiometric imaging and quantitative comparison of the fluorescence intensities within the drug-treated and non-treated control samples. The images were 1024x1024 pixels and the pixel size was $103.6 \mu \mathrm{m}$ in $\mathrm{x} / \mathrm{y}$. The images were acquired without averaging and by first focusing on the bottom surface of the sample, where the position of the sample stage was set as $z 0=0$. The fluorescence signal intensities from all emission channels were then collected from bottom to top as optical z-series with $200 \mathrm{~nm}$ step size. The pinhole was set to 0.9 (physical pinhole size $34.76 \mu \mathrm{m}$ ). The analysis was done in Image J software by making maximum intensity projections from the acquired $\mathrm{z}$ stacks, and by using the LA/C-rod channel to segment the nuclei which was then used as a mask to measure the maximum signal intensities for all channels. The mean intensities of the nuclei, the 
background, and the total images were determined. To detect changes in the lamin organization the nuclear lamin intensity ratio (LA/C-C:LA/C-rod) was calculated from the nuclear intensities of which the detector noise was subtracted and which were normalized against their background. Number of replicates, $\mathrm{n}=3$ for all treatments. Unpaired Student's t-test was used to test the statistical significance between treated and non-treated control samples. ns $=$ non-significant, $* \mathrm{p}<0.05, * * \mathrm{p}<0.01, * * * \mathrm{p}<0.001$.

\section{Super-resolution Airy-scan Imaging}

Zeiss LSM 980 laser scanning confocal microscope with airyscan was used for fixed-cell experiments. The system was mounted on Axio Observer.Z1 microscope body and PlanApochromat 63X/1.4 oil immersion objective was used in the imaging. The sensor and the immunolabelled lamin A/C were excited with $488 \mathrm{~nm}$ and $639 \mathrm{~nm}$ lasers using MDS488/561/639 triple dicroic and the emission was collected with band-pass 495-560 nm and long-pass $650 \mathrm{~nm}$ filters. The image size was set to 1032 x 1032 pixels, with pixel size of $43 \mathrm{~nm}$ and optical section collected with $170 \mathrm{~nm}$ intervals. Scanning was bidirectional with $2 \mu$ s pixel dwell time and averaging of 4 was used. Data was analysed with ImageJ FIJI -distribution.

\section{Fluorescence Recovery After Photobleaching (FRAP)-Experiments}

Zeiss LMS780 laser scanning confocal microscope in inverted Cell observer microscope body was used in the experiments. MDCK cells stably expressing Lamin-SS or Lamin-SS-T were seeded on collagen-I -coated (50 $\mu \mathrm{g} / \mathrm{mL}$ in PBS, $45 \mathrm{~min}$ in RT) high performance coverslips (Zeiss, \#4740309020-000) $1 \mathrm{~d}$ before the experiments. Prior imaging, the coverslips were mounted on imaging chamber (Aireka Cells, \#SC15022, Aireka Scientific, HK, China) and placed in the microscope incubator $\left(37{ }^{\circ} \mathrm{C}, 5 \% \mathrm{CO}_{2}\right)$. Imaging was conducted by using $63 \mathrm{X} / 1.2 \mathrm{WI} \mathrm{C}$-Apochormat objective. Lamin-SS or Lamin-SS-T was excited with $514 \mathrm{~nm}$ laser line, pixel size was adjusted to $0.13 \mu \mathrm{m}$ (zoom setting 4) and 256 x 256-pixel images were captured without averaging (195 ms scanning time per frame). In the FRAP experiment, images were collected with $250 \mathrm{~ms}$ intervals (249 images altogether), and a bleaching was conducted after 9 scans. In the bleaching phase, a pre-drawn rectangular area of $75 \times 10$ pixels in the nuclear lamina was scanned 25 times (iterations) with $100 \%$ light intensity from $514 \mathrm{~nm}$ laser. The recovery was then followed for 240 frames.

\section{FRAP Data Analysis and Simulations}

FRAP recovery curves were measured by using ImageJ FIJI-distribution[19]. The drift of the nucleus during the imaging was corrected by using StackReg-plugin[20]. Next the fluorescence 
was measured from the lamina and from the whole nucleus. The data was then normalized in Microsoft Excel for Mac (version 16.55) according to Phair \& Misteli[21]:

$I(t)=(\operatorname{lamina}(t) / \operatorname{lamina}(t=0)) /(\operatorname{nucleus}(t) / \operatorname{nucleus}(t=0))$

Where lamina $(\mathrm{t})$ is fluorescence in the lamina at time point $\mathrm{t}$, lamina $(\mathrm{t}=0)$ is fluorescence in the lamina before the bleach phase, nucleus $(\mathrm{t})$ is the fluorescence of the whole nucleus at time point $\mathrm{t}$ and nucleus $(t=0)$ is the fluorescence of the nucleus before the the bleach phase. Finally, the normalized recoveries were averaged.

Virtual Cell software[22], [23]was used to simulate the FRAP experiment and fluorescence recovery. The model contains a free Lamin-SS sensor which can bind to an immobile binding site in the lamina (single bound sensor), this binding can then lead into release of the sensor or tighter binding, simulating the situation where the sensor is engaged from both nanobodies (dual bound sensor). The release of the dual bound sensor was assumed to happen via single bound-state. The Lamin-SS-T sensor behavior was assumed to behave otherwise similarly, only the dual binding opportunity was missing. The reaction network schematic is visualized in Supplementary Figure 2. The Virtual Cell Models, "Lamin-SS_dual_binding" and "Lamin-SS-T_single_binding” by user "teihalai", can be freely accessed within the VCell software (available at https://vcell.org).

\section{SensorFRET Imaging and Analysis}

Live cells were seeded on glass-bottom slides coated with $20 \mu \mathrm{g} / \mathrm{mL}$ fibronectin. DMEM was replaced with live cell imaging solution (cat \#: A14291DJ, Thermo Fisher) supplemented with 10\% FBS. Images were acquired using an inverted Zeiss LSM 710 (Oberkochen, Germany) confocal microscope using both $405 \mathrm{~nm}$ or $458 \mathrm{~nm}$ excitation wavelengths from an argon laser source. A 40x water immersion objective lens $(\mathrm{NA}=1.1)$ was used for all imaging. Live cells were imaged in spectral mode using a 32-channel spectral META detector to record spectra of each pixel spanning wavelengths from 416 to $718 \mathrm{~nm}$ (with $9.7 \mathrm{~nm}$ spectral steps). Images were captured in 16-bit mode, scanned bi-directionally, and averaged 4 times. For sensorFRET based efficiency imaging, spectral images at both 405 and $458 \mathrm{~nm}$ excitation wavelengths were acquired. The normalized emission shape of the mTFP and mVenus fluorophores as well as the calibration parameter $\mathrm{c}(=0.101)$ required for the sensorFRET analysis were experimentally determined from control cells expressing single fluorophores[24]. Intensity images were further processed and analyzed using a custom Python code, which involves background subtraction and removal of saturated pixels. For each data set, the data was acquired for at least 5 images per condition per experiment. Images were masked manually on Fiji Image J. 


\section{Paired FRET Measurements and Analysis}

Ratiometric FRET imaging was used for FRET measurements involving paired FRET samples. Cell seeding and mounting was performed with similar protocol as in FRAP experiments. For live cell imaging cells were placed in the microscope incubator $\left(37^{\circ} \mathrm{C}, 5 \% \mathrm{CO}_{2}\right)$. Zeiss LSM 780 laser scanning confocal microscope equipped with Plan Apochromat 63x/1.4 oil immersion objective was used for ratiometric FRET approach. FRET imaging and analysis was done by RiFRET method described previously[25]. Briefly, the donor and acceptor were excited with a $458 \mathrm{~nm}$ line and a $514 \mathrm{~nm}$ line, respectively, from a multiline argon laser. The resulting fluorescence was acquired between 465-500 $\mathrm{nm}$ for donor emission and 535-650 $\mathrm{nm}$ for acceptor emission with a 32-channel QUASAR GaAsP PMT array detector. FRET channel emission was obtained with donor excitation $(458 \mathrm{~nm})$ and detected through the acceptor emission channel. Cells stably expressing either donor or acceptor probes alone was used to determine the spectral cross-talk. RiFRET plugin[25] for ImageJ was used for cross-talk correction of each channel and to calculate pixel by pixel-based apparent FRET efficiency. The apparent FRET efficiency from individual cells prior to and after treatment was used for analysis.

\section{Fluorescence Lifetime Imaging Microscopy (FLIM) -FRET analysis}

For FLIM, cells cultured in coverslips were fixed with 4\% PFA for 10 mins, washed and stored in PBS at $4{ }^{\circ} \mathrm{C}$ in dark before imaging. Prior imaging, the coverslips were mounted on imaging chamber and PBS was added to the chamber. Fluorescence lifetime imaging was performed using Leica STELLARIS FALCON confocal microscope equipped with Plan Apochromat 40x/1.25 motCORR glycerol immersion objective. Cells were excited with White Light Laser Stellaris 8 at $450 \mathrm{~nm}$, and fluorescence lifetime times were recorded with HyD X detector, in the range 455 to $495 \mathrm{~nm}$ to obtain the photon arrival times specific to donor emission. The pixel-bypixel photon arrival times were fitted for bi-exponential decay components using n-Exponential Reconvolution fitting model of Leica LAS X software to obtain mean lifetimes from individual cells.

\section{Statistical Analysis}

Statistical significance was measured using an unpaired, two-tailed Student's t-test for data containing two groups. For data involving more than two groups, the Ordinary One-way Analysis of Variance (ANOVA) test was performed in order to obtain the statistical analysis for the data sets concerned. A further comparison of the groups was conducted using the Tukey (HSD) test so as to obtain significant differences between multiple groups. All statistical tests were conducted at a 5\% significance level. Prism Graphpad was used for statistical analyses. 


\section{Supplemental Figure legends}

\section{Supplemental Figure 1. Fluorescence recovery after photobleaching (FRAP) experiments of Lamin-SS and Lamin-SS-T binding to nuclear lamina.}

a, Model of the Lamin-SS and Lamin-SS-T binding to nuclear lamina. Binding of Lamin-SS is assumed to proceed sequentially, from the binding of the first nanobody (single site) to the binding of the other (dual site). The release of the sensor from the lamina is modeled to proceed in reverse. Lamin-SS-T binding is limited to single site binding. b, FRAP experiment with Lamin-SS expressing cell. Bleached region of interest (ROI) is marked in the blowup image. The initial recovery is rapid, indicating fast binding dynamics. Scale bar $5 \mu \mathrm{m}$. c, Quantified and normalized fluorescence recoveries (mean \pm standard deviation) of Lamin-SS and Lamin-SS-T indicating difference in the recovery dynamics ( $n=19$ and $n=18$ cells, respectively, from 2 replicates). d, Simulated recovery data together with the measured recoveries, see also supplemental figure 2. e, Binding pseudo on-rate and off-rate of Lamin-SS and Lamin-SS-T used in the simulations shown in d. f, Lamin-SS and Lamin-SS-T binding times and corresponding fractions based on the simulated recoveries.

\section{Supplemental Figure 2. Schematic representation of the simulated FRAP experiment and reactions.}

a, Reactions of Lamins-SS during FRAP experiment. Freely diffusing Lamin-SS interacts with a binding site in the nuclear lamina (single site binding reaction) yielding Single bound Lamin-SS. This can further lead into Dual bound Lamin-SS (single-to-dual site binding reaction) or release of the sensor and binding site (reverse of single site binding reaction). Dual bound Lamin-SS can be released by reverse reaction leading into single bound Lamin-SS (reverse of single-to-dual site binding reaction). Bleaching is simulated by a local reaction between Bleaching laser, and free Lamin-SS, Single bound Lamin-SS and Dual bound Lamin-SS. The bleaching leads into appearance of bleached species of Lamin-SS. b, Reactions of Lamins-SS-T during FRAP experiment. Truncated sensor binding is limited to the reaction between free Lamin-SS-T and binding site in the lamina, yielding Single bound Lamin-SS-T (single site binding reaction). Similarly, as Lamin-SS, truncated Lamin-SS-T sensor is released, leading to Free Lamin-SS-T and Binding site in the lamina (reverse of Single site binding reaction). Fluorescent Lamin-SS-T molecules are bleached in the Bleaching reactions with Bleaching laser, yielding Bleached single bound Lamin-SS-T and Bleached free Lamin-SS-T. 
Supplemental Figure 3. Lamin-SS, Lamin-histone-SS and Lamin-histone-SS-T sensor distribution and apparent FRET efficiency in LMNA KO cells.

a, Wild-type (WT) and LMNA KO cells transiently transfected with Lamin-SS. In LMNA KO cells Lamin-SS localization to nuclear lamina is lost. b, WT and LMNA KO cells transiently transfected with Lamin-histone-SS. c, WT cells transiently transfected with Lamin-histone-SS-T. Scale bars $10 \mu \mathrm{m}$. d, Quantified apparent FRET efficiency (mean \pm SEM) of Lamin-SS and Laminhistone-SS in WT and LMNA KO cells, and apparent FRET efficiency of Lamin-histone-SS-T in WT cells. Lamin-SS FRET was $0.151 \pm 0.010$ in WT and $0.209 \pm 0.009$ in LMNA KO cells ( $n=31$ and $n=22$, respectively, 2 replicates) $(* * * p=0.0001)$. Lamin-histone-SS FRET was $0.200 \pm 0.009$ in WT and $0.239 \pm 0.008$ in LMNA KO cells $(n=38$ and $n=40$, respectively, 1 replicate) $(* *$ $\mathrm{p}=0.003)$ Lamin-histone-SS-T FRET was $0.272 \pm 0.003$ in WT cells $(\mathrm{n}=38,1$ replicate) $(* * *$ $\mathrm{p}=0.0008$, when compared to Lamin-histone-SS in KO cells). Unpaired Student's t-tests.

\section{Supplemental Figure 4. Effect of hyper-osmotic conditions on nuclear morphology and volume.}

a, Lamin-SS expressing cells (left) were subjected to hyper-osmotic conditions by adding media containing $250 \mathrm{mM}$ concentration of sucrose for $15 \mathrm{~min}$ (middle). Single cell blow-up indicated the change in nuclear morphology before and after the osmotic shock (right). Scale bar $10 \mu \mathrm{m}$. b, Scatterplot of quantified nuclei volumes indicating clear reduction of the nuclear volume.

\section{Supplemental Figure 5. Development and characterization of the FRET based lamin A/C - histone H2A strain sensor.}

a, Schematic representation of the FRET based lamin A/C - histone H2A strain sensor (Laminhistone-SS), truncated control sensor (Lamin-histone-SS-T) and the working mechanism of the force sensing between lamina and chromatin. b, Laser scanning confocal microscopy images (Airy-scan, single sections) of immunolabeled lamin A/C, histone H2A and the expressed Laminhistone-SS sensor along with corresponding fluorescence line-profiles. Scale bar $5 \mu \mathrm{m}$. c, FRET efficiency images and quantified FRET efficiency of Lamin-histone-SS and Lamin-histone-SS-T sensors. The plots represent the median \pm SEM of individual image fields. Lamin-histone-SS had a median FRET efficiency of $17 \%$ compared to Lamin-histone-SS-T with $40 \%$ (total $n=10$ from 3 replicates). Scale bar $20 \mu \mathrm{m}$. Unpaired Student's t-test $(* \mathrm{p}<0.05)$.

\section{Supplemental Figure 6. The effect of actin cytoskeleton disruption on Lamin-SS FRET}

Live cell imaging of Lamin-SS apparent FRET ratio during actin cytoskeleton disruption by Cytochalasin D $(10 \mu \mathrm{g} / \mathrm{mL})$. Scale bar $5 \mu \mathrm{m}$. Blow-up images of single nucleus indicate increase 
in the FRET. Quantified apparent FRET ratio of the nuclei before and after Cytochalasin D treatment (left). The median FRET ratio increased from $4.3 \%$ before to $5.1 \%$ after the treatment $(n=92$ cells, from 2 biological replicates). Student's paired t-test $(* * p<0.01)$.

\section{Supplemental Figure 7. Nuclear lamina organization after chromatin relaxation}

a, Laser scanning confocal microscopy maximum intensity projection images of control (upper panels) and trichostatin A (TSA) -treated (600 nM, 4 h, lower panels) Lamin-SS expressing cells, immunolabeled against histone H3 lysine 27 acetylation (H3K27) and N-terminal part of A-type lamins. Scale bars, $10 \mu \mathrm{m}$. b, Quantification of nuclear fluorescence intensity of H3K27 acetylation labeling in control and TSA-treated cells $(n=159$ and $n=151$ cells, respectively, from 3 biological replicates). In control cells the fluorescence intensity was $877.62 \pm 556.12$ artificial units (a.u.) (mean \pm standard deviation) and after TSA treatment $1527.06 \pm 584.22$ a.u. Unpaired Student's t-test (** $\mathrm{p}=0.004$ ). c, Quantification of nuclear fluorescence intensity of N-terminal nuclear lamin A/C label in control and TSA-treated cells ( $n=159$ and $n=151$ cells, respectively, from 3 biological replicates). In control cells the fluorescence intensity was $1774.31 \pm 951.68$ a.u. and after the TSA treatment $1984.70 \pm 621.638$ a.u. Unpaired Student's t-test $(\mathrm{p}=0.5$, nonsignificant (ns)). d, Laser scanning confocal microscopy maximum intensity projection images of control (upper panels) and TSA-treated (600 nM, 4 h, lower panels) Lamin-SS expressing cells, immunolabeled against C-terminal part and rod-domain of A-type lamins. Scale bars, $10 \mu \mathrm{m}$. e, Quantified fluorescence intensity ratio of lamin $\mathrm{A} / \mathrm{C} \mathrm{C}$-terminus and rod-domain labeling in control and TSA-treated cells $(n=291$ and $n=276$ cells, respectively, from, 3 biological replicates). In control cells the ratio was $0.72 \pm 0.30$ and in the TSA treated cells $0.69 \pm 0.18$. Unpaired Student's t-test, $(\mathrm{p}=0.6, \mathrm{~ns})$.

\section{Supplemental Figure 8. Fluorescence recovery after photobleaching (FRAP) experiments of Lamin-SS after TSA treatment.}

a, FRAP experiment with Lamin-SS expressing cells in control (upper panel) and after TSA treatment $(600 \mathrm{nM}, 4 \mathrm{~h})$. Bleached region of interest (ROI) is marked in the image. Scale bar $5 \mu \mathrm{m}$. b, Quantified and normalized fluorescence recoveries (mean \pm standard deviation) of Lamin-SS in control and TSA treated cells ( $n=10$ and $n=13$, respectively, from 2 biological replicates).

\section{Supplemental figure 9. Nuclear lamina organization after chromatin condensation}

a, Laser scanning confocal microscopy maximum intensity projection images of control (upper panels) and methylstat -treated $(2.5 \mu \mathrm{M}, 48 \mathrm{~h}$, lower panels) Lamin-SS expressing cells, 
immunolabeled against lamin $\mathrm{A} / \mathrm{C} \mathrm{C}$-terminus and rod-domain Scale bars, $10 \mu \mathrm{m} . \mathbf{b}$, Quantification of nuclear fluorescence intensity of lamin A/C C-terminus labeling in control and methylstat-treated cells ( $n=244$ and $n=251$ cells, respectively, from 3 biological replicates). In control cells the fluorescence intensity was $992.77 \pm 324.17$ a.u. and after methylstat treatment $1553.69 \pm 397.16$ a.u. Unpaired Student's unpaired t-test (*** $\mathrm{p}=0.0002)$. c, Quantification of nuclear fluorescence intensity of lamin $\mathrm{A} / \mathrm{C}$ rod-domain labeling in control and methylstat-treated cells ( $n=244$ and $n=251$ cells, respectively, from 3 biological replicates). In control cells the fluorescence intensity was $871.24 \pm 275.77$ a.u. and after methylstat treatment $1296.36 \pm 299.66$ a.u. Unpaired Student's t-test (*** $\mathrm{p}=0.0004)$. d, Quantified fluorescence intensity ratio of lamin A/C C-terminus and rod-domain labeling ( $n=244$ and $n=251$ cells, respectively, from 3 biological replicates). In control cells the ratio was $1.14 \pm 0.13$ and in the methylstat-treated cells $1.22 \pm 0.33$. Unpaired Student's t-test, $\mathrm{p}=0.37$, ns.

\section{References}

[1] P. Roca-Cusachs, V. Conte, and X. Trepat, "Quantifying forces in cell biology," Nat. Cell Biol. 2017 197, vol. 19, no. 7, pp. 742-751, Jun. 2017.

[2] A. Tajik et al., "Transcription upregulation via force-induced direct stretching of chromatin," Nat. Mater., vol. 15, no. 12, pp. 1287-1296, 2016.

[3] T. J. Kirby and J. Lammerding, "Emerging views of the nucleus as a cellular mechanosensor,” Nat. Cell Biol., vol. 20, no. 4, pp. 373-381, 2018.

[4] S. Osmanagic-Myers, T. Dechat, and R. Foisner, "Lamins at the crossroads of mechanosignaling," Genes and Development, vol. 29, no. 3. Cold Spring Harbor Laboratory Press, pp. 225-237, 2015.

[5] T. Dechat et al., "Nuclear lamins: major factors in the structural organization and function of the nucleus and chromatin," Genes Dev., vol. 22, no. 7, pp. 832-853, Apr. 2008.

[6] Y. Turgay et al., "The molecular architecture of lamins in somatic cells," Nature, vol. 543, no. 7644, pp. 261-264, Mar. 2017.

[7] N. Naetar, S. Ferraioli, and R. Foisner, "Lamins in the nuclear interior - life outside the lamina," J. Cell Sci., vol. 130, no. 13, pp. 2087-2096, Jul. 2017.

[8] K. T. Sapra et al., "Nonlinear mechanics of lamin filaments and the meshwork topology build an emergent nuclear lamina," Nat. Commun. 2020 111, vol. 11, no. 1, pp. 1-14, Dec. 2020. 
[9] C. Grashoff et al., "Measuring mechanical tension across vinculin reveals regulation of focal adhesion dynamics," Nat. 2010 4667303, vol. 466, no. 7303, pp. 263-266, Jul. 2010.

[10] P. T. Arsenovic et al., "Nesprin-2G, a Component of the Nuclear LINC Complex, Is Subject to Myosin-Dependent Tension,” Biophys. J., vol. 110, no. 1, pp. 34-43, Jan. 2016.

[11] S. J. Tan et al., "Regulation and dynamics of force transmission at individual cell-matrix adhesion bonds," Sci. Adv., vol. 6, no. 20, May 2020.

[12] A. Kumar et al., "Talin tension sensor reveals novel features of focal adhesion force transmission and mechanosensitivity," J. Cell Biol., vol. 213, no. 3, pp. 371-383, May 2016.

[13] C. Grashoff et al., "Measuring mechanical tension across vinculin reveals regulation of focal adhesion dynamics," Nature, vol. 466, no. 7303, pp. 263-266, Jul. 2010.

[14] U. Rothbauer et al., "Targeting and tracing antigens in live cells with fluorescent nanobodies," Nat. Methods, vol. 3, no. 11, pp. 887-889, Nov. 2006.

[15] C. Grashoff et al., "Measuring mechanical tension across vinculin reveals regulation of focal adhesion dynamics," Nature, vol. 466, no. 7303, pp. 263-266, Jul. 2010.

[16] D. Jullien et al., "Chromatibody, a novel non-invasive molecular tool to explore and manipulate chromatin in living cells," J. Cell Sci., vol. 129, no. 13, pp. 2673-2683, Jul. 2016.

[17] Q. Zhang et al., "Mechanical Stabilization of the Glandular Acinus by Linker of Nucleoskeleton and Cytoskeleton Complex," Curr. Biol., vol. 29, no. 17, pp. 28262839.e4, Sep. 2019.

[18] D. A. Nelles et al., "Programmable RNA Tracking in Live Cells with CRISPR/Cas9," Cell, vol. 165, no. 2, pp. 488-496, Apr. 2016.

[19] J. Schindelin et al., "Fiji: an open-source platform for biological-image analysis," Nat. Methods 2012 97, vol. 9, no. 7, pp. 676-682, Jun. 2012.

[20] P. Thévenaz, U. E. Ruttimann, and M. Unser, "A pyramid approach to subpixel registration based on intensity," IEEE Trans. Image Process., vol. 7, no. 1, pp. 27-41, 1998.

[21] R. D. Phair and T. Misteli, "High mobility of proteins in the mammalian cell nucleus," Nat. 2000 4046778, vol. 404, no. 6778, pp. 604-609, Apr. 2000.

[22] D. C. Resasco, F. Gao, F. Morgan, I. L. Novak, J. C. Schaff, and B. M. Slepchenko, "Virtual Cell: computational tools for modeling in cell biology," Wiley Interdiscip. Rev. Syst. Biol. Med., vol. 4, no. 2, p. 129, Mar. 2012.

[23] J. Schaff, C. C. Fink, B. Slepchenko, J. H. Carson, and L. M. Loew, “A general computational framework for modeling cellular structure and function.," Biophys. J., vol. 73, no. 3, p. 1135, 1997.

[24] P. T. Arsenovic, C. R. Mayer, and D. E. Conway, "SensorFRET: A Standardless Approach to Measuring Pixel-based Spectral Bleed-through and FRET Efficiency using 
bioRxiv preprint doi: https://doi.org/10.1101/2022.03.07.483300; this version posted March 7, 2022. The copyright holder for this preprint (which was not certified by peer review) is the author/funder. All rights reserved. No reuse allowed without permission.

Spectral Imaging,” Sci. Rep., vol. 7, no. 1, pp. 1-15, Dec. 2017.

[25] J. Roszik, D. Lisboa, J. Szöllosi, and G. Vereb, "Evaluation of intensity-based ratiometric FRET in image cytometry-Approaches and a software solution," Cytom. Part A, vol. 75A, no. 9, pp. 761-767, Sep. 2009. 
a

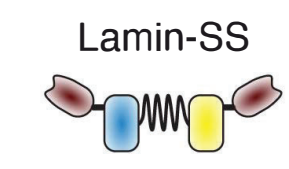

0 chromin

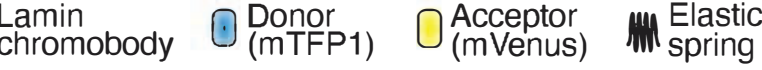

\section{Low strain}
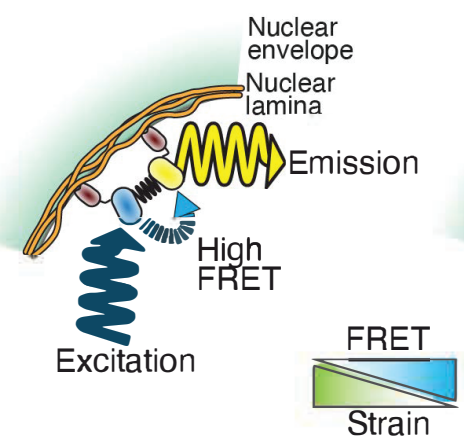

Lamin-SS-T

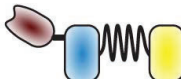

High strain

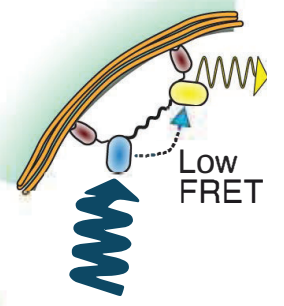

b

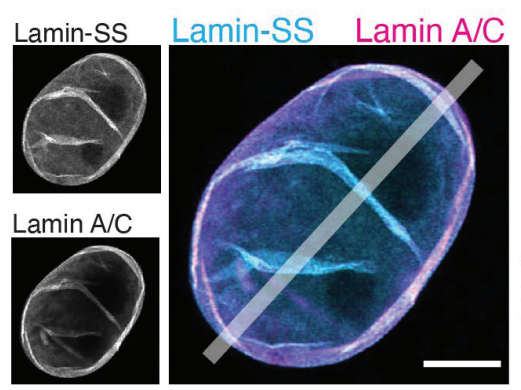

Lamin-SS-T Lamin-SS-T Lamin A/C
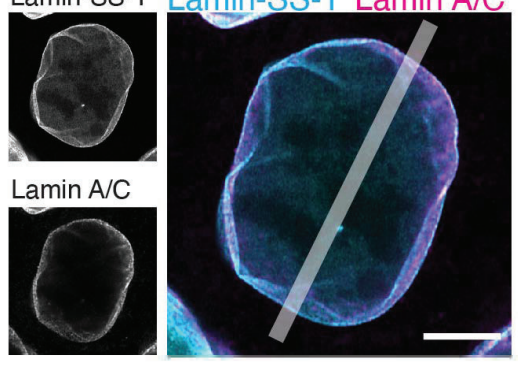

Profile

Lamin A/C

Lamin-SS

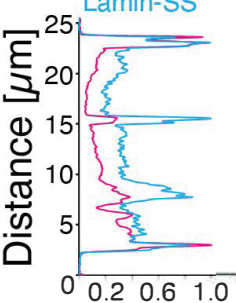

Fluorescence

[a.u.]

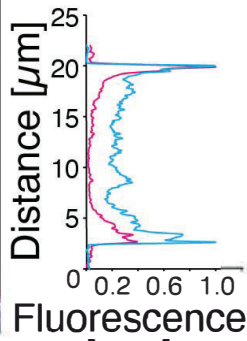

[a.u.]

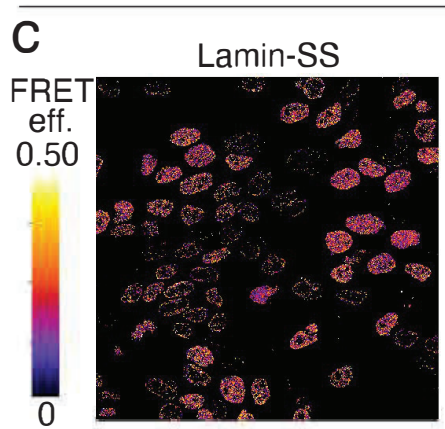

Lamin-SS-T

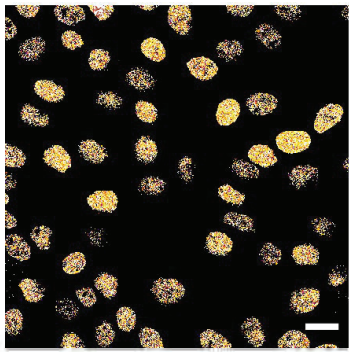

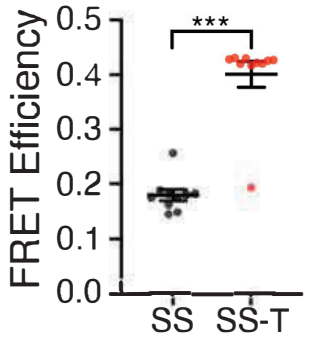
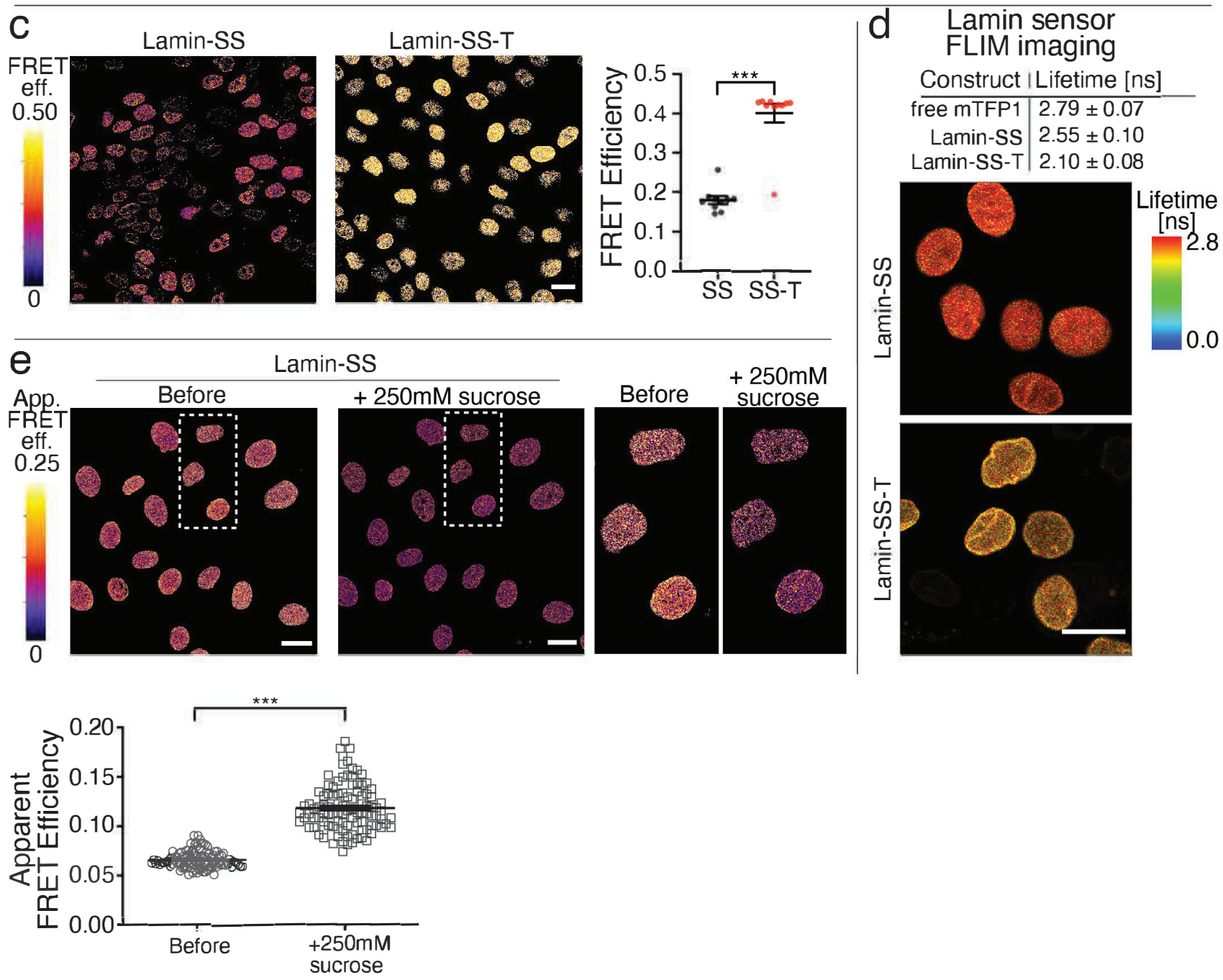
Figure tyich was not certified by peer review) is the author/funder. All rights reserved. No reuse allowed without permission.

a

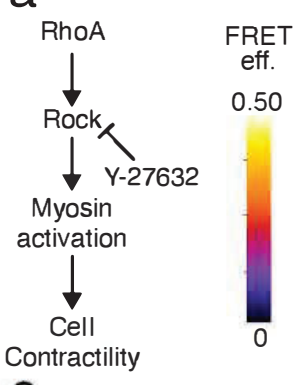

C

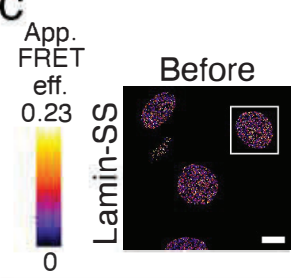

Control

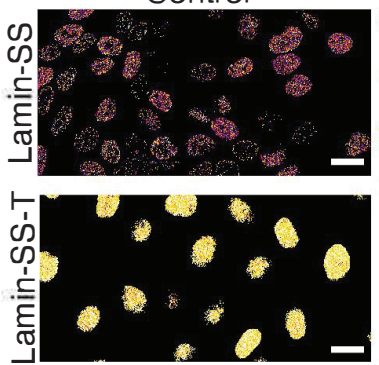

Y-27632

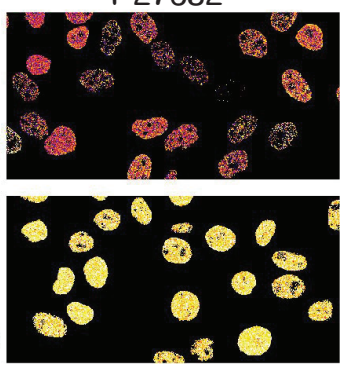

Y-27632

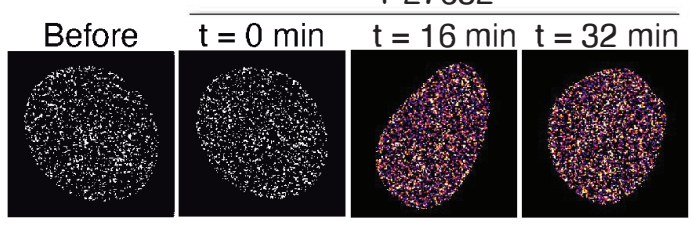

b

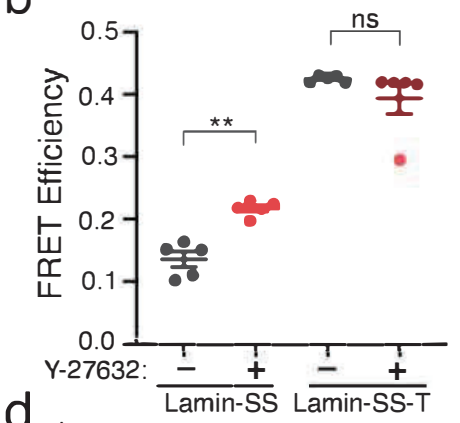

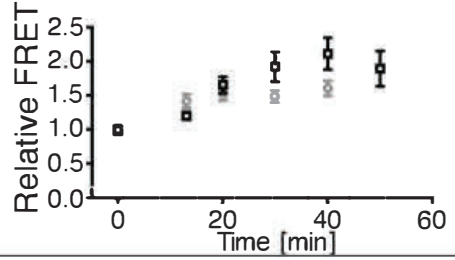

e LINCcomplexes

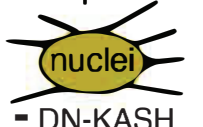

- DN-KASH
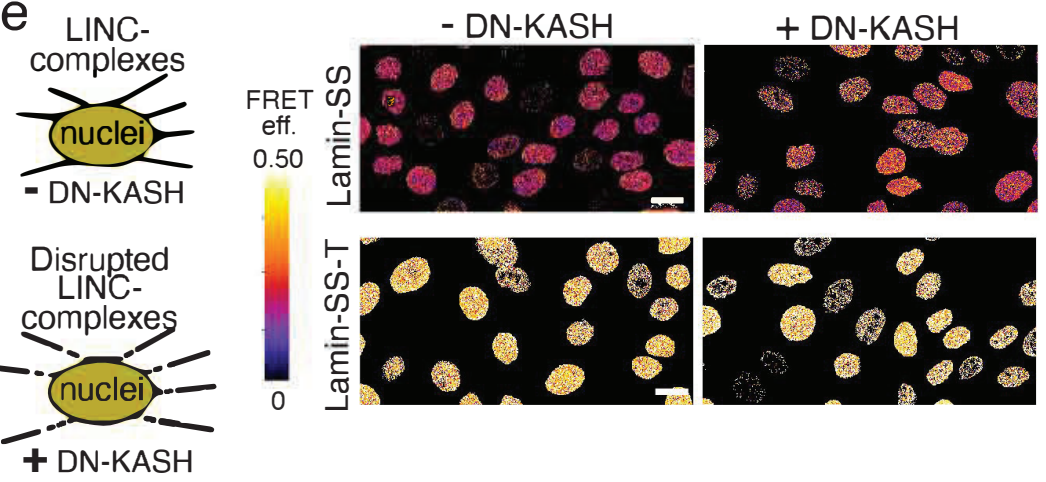

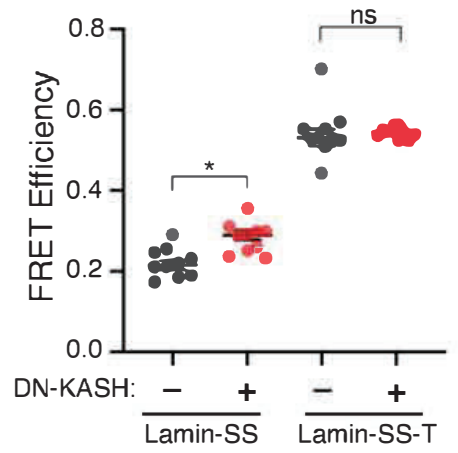

g Chromatin relaxation

Control TSA

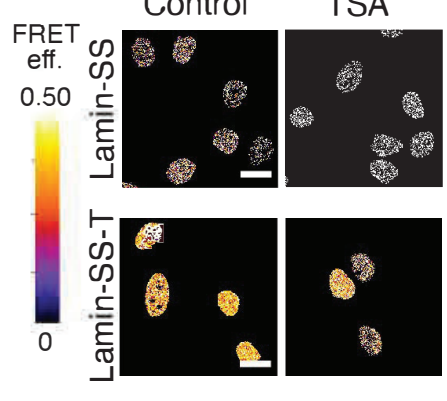

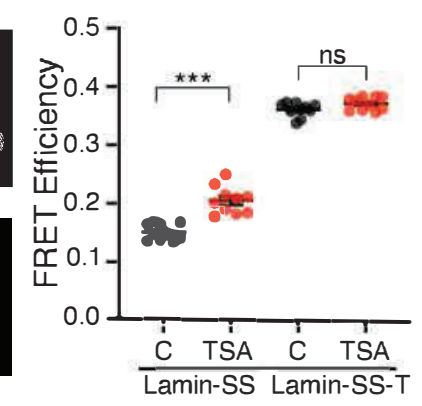

h Chromatin compaction
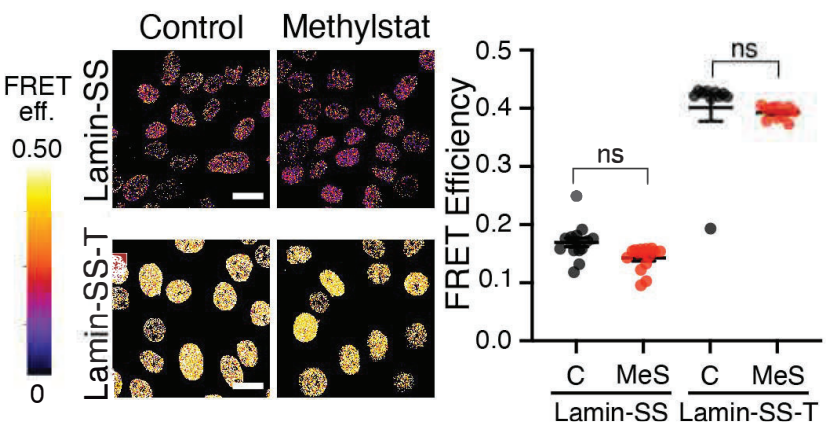

i Cell cycle $\underset{\text { Aphidicolin }}{\longrightarrow} \underset{\text { S }}{\longrightarrow}$
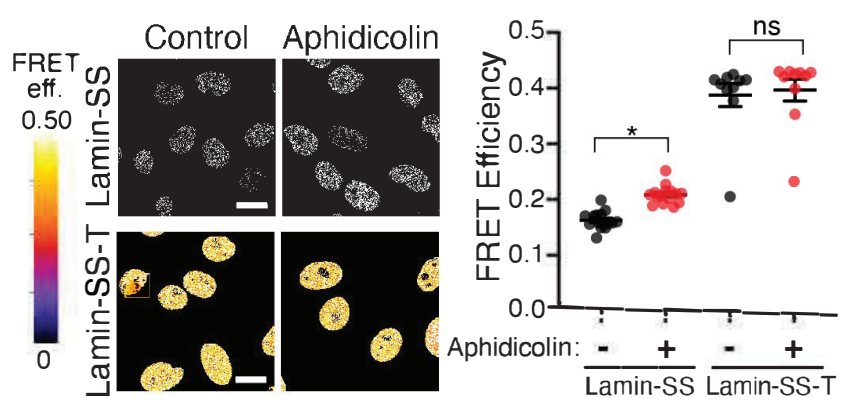

j
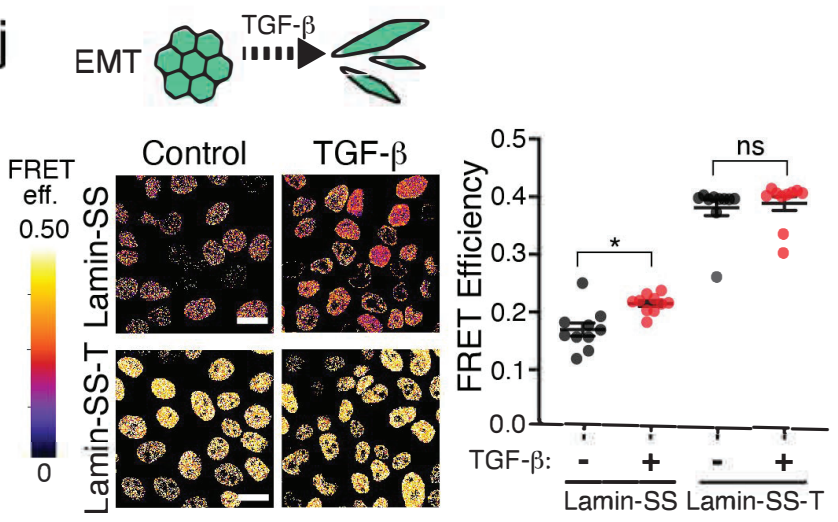